\title{
Assessment by Thallium-201 of Hindlimb Muscle Blood Flow in Rats during Recovery after Hindlimb Unloading
}

\author{
Pleiades TiHARU INAOKA ${ }^{1)}$, RYOHEI AMANO ${ }^{2)}$, SHOJI TANAKA ${ }^{1)}$, KATSUHIKO TACHINO ${ }^{1)}$ \\ 1) Department of Impairment Study, Graduate Course of Rehabilitation Science, Division of \\ Health Sciences, Graduate School of Medical Science, Kanazawa University: 5-11-80 \\ Kodatsuno, Kanazawa, Ishikawa 920-0942, Japan. \\ TEL: +81 76-265-2616, E-mail: inaoka@mhs.mp.kanazawa-u.ac.jp \\ ${ }^{2)}$ Department of Forefront Medical Technology, Graduate Course of Medical Science and \\ Technology, Division of Health Sciences, Graduate School of Medical Science, Kanazawa \\ University
}

\begin{abstract}
Purpose] We aimed to evaluate the muscle blood perfusion by the uptake of the radioisotope thallium-201 $\left({ }^{201} \mathrm{Tl}\right)$ in 5 hindlimb muscles, the soleus (Sol), plantaris (Pla), gastrocnemius (Gas), extensor digitorum longus (EDL), and tibialis anterior (TA). [Subjects] The subjects were 36 male Wistar rats. [Methods] ${ }^{201} \mathrm{Tl}$ uptake rates were calculated by spectrometry and visualized by autoradiography (ARG) in hindlimb unloading $(\mathrm{U})$ and reloading $(\mathrm{R})$ rats. [Results] The Sol ${ }^{201} \mathrm{Tl}$ uptake rates were significantly lower in $U$ groups $(p<0.01)$ than in the control group. Meanwhile the reloading day-4 group had higher values in Sol than the control $(p<0.01)$. In $U$ groups, the EDL and TA uptake rates were higher than in the other 3 muscles (Sol, Pla, and Gas). [Conclusion] We concluded that during 21 days of hindlimb unloading and subsequent recovery by reloading, blood capillary perfusion assessed by ${ }^{201} \mathrm{Tl}$ in hindlimb muscles changes in rats. The results confirm the utility of ${ }^{201} \mathrm{Tl}$ in quantitative and qualitative evaluation of skeletal muscle activity and blood perfusion, which has applications in rehabilitation and sports science.
\end{abstract}

Key words: Hindlimb reloading, Thallium-201, Blood flow

(This article was submitted Sep. 9, 2008, and was accepted Jan. 13, 2009)

\section{INTRODUCTION}

Thallium-201 $\left({ }^{201} \mathrm{Tl}\right)$ is a radioisotope available commercially as thallous chloride $\left({ }^{201} \mathrm{TlCl}\right)$. In its monovalent form, thallium behaves like an alkali metal ion, similar to potassium; therefore it enters the cells by similar transport mechanisms after injection. The ${ }^{201} \mathrm{Tl}$ fast uptake velocity initially provides visualization of organ and tissues according to blood flow. The delay images after represent the redistribution of the tracer and can reveal viable areas of damaged tissue ${ }^{3)}$. The thallium have wide clinical application, including myocardial visualization and monitoring of leg perfusion, tumor uptake, and thyroid uptake ${ }^{1-5)}$. The most common use of ${ }^{201} \mathrm{Tl}$ is in cardiac imaging. It can be used to assess not only myocardial perfusion, but also myocyte cell membrane integrity.

Disuse atrophy is a reduction in muscle volume generally accompanied by muscle weakness that is frequently seen in clinical situations. To date, no effective method has been established for the prevention of disuse muscle atrophy ${ }^{6}$. Although the mechanism is unclear, disuse atrophy affects slowtwitch fibers (Type I) to a greater extent than fasttwitch fibers (Type II) ${ }^{7)}$. The soleus (Sol) and 
extensor digitorum longus (EDL) are frequently studied because they are considered representative of slow-twitch and fast-twitch muscles, respectively, due to their main fiber composition.

We examined the ${ }^{201} \mathrm{Tl}$ uptake behavior in 5 hindlimb muscles of rats, including the soleus and EDL, to examine the blood flow distribution among the muscles in hindlimb unloading (HU) and reloading $(\mathrm{R})$ in model rats. Our previous study revealed different patterns in the blood flow on these muscles which were dependent on the time from ${ }^{201} \mathrm{Tl}$ injection, state of consciousness and the muscular trophicity ${ }^{9}$. Mizuno ${ }^{10)}$ concluded that muscle blood flow and metabolism remain matched during rest. The ${ }^{201} \mathrm{Tl}$ blood flow evaluation method of the present study may lead to the development of a noninvasive assessment of muscle metabolism, which would be useful in the evaluation of the effectiveness of rehabilitation treatment for muscle pathologies.

\section{SUBJECTS AND METHODS}

\section{Subjects}

All animal experiments were carried out in compliance with the guidelines for the care and use of laboratory animals and were approved by the Committee on Animal Experimentation of Kanazawa University.

Thirty six male Wistar rats, initially aged 8 weeks old (body weight $=240 \sim 260 \mathrm{~g}$ ), were purchased from Charles River Japan Inc and housed one per cage in a temperature-controlled $\left(20 \sim 24{ }^{\circ} \mathrm{C}\right)$ room with a 12-h light-dark cycle. Water and standard laboratory chow were provided ad libitum.

The rats were subjected to hindlimb unweighting (HU) for 3 weeks, as described by Yamazaki et al. ${ }^{8}$. They were able to move with their forelimbs and had access to food and water ad libitum.

The experimental animals were randomly assigned to reloading groups $(\mathrm{R})$ which were reloaded after 3 weeks of unloading. The unloaded groups (U) consisted of 3-week-unloaded group (U3w) and the group at the start of hindlimb unloading (UØ). The reloaded groups were subjected to 10 minutes (R10m), 6 hours (R6h), 24 hours (R24h), 4 days (R4d), and 12 days (R12d) of reloading following HU. Control groups consisted of normal caged rats assigned to age-matched Control 1 (C1, age-matched to UØ, U3w, R10m, R6h, R24h and R4d groups) and age-matched Control 2 (C2, age-matched to R12d) groups. All animals were sacrificed 30 min after ${ }^{201} \mathrm{TlCl}$ injection.

\section{Methods}

Commercial ${ }^{201} \mathrm{TlCl}$ was diluted in physiological saline solution to prepare the radioactive stock solution. Under diethyl ether anesthesia $0.1 \mathrm{ml}$ of the solution $(5 \mathrm{MBq})$ was injected intraperitoneally. A preliminary experiment was performed to investigate ${ }^{201} \mathrm{Tl}$ kinetics in rat muscles and determine the appropriate timing of tissue removal $^{9)}$. Thirty minutes after ${ }^{201} \mathrm{TlCl}$ injection the animals were sacrificed by excessive inhalation of diethyl ether for immediate tissue harvesting.

Samples of blood and the five hindlimb muscles-i.e., the soleus (Sol), plantaris (Pla), medial head of the gastrocnemius (Gas), extensor digitorum longus (EDL), and tibialis anterior (TA) - were removed in sequence. The tissues were weighed immediately and divided in two for spectrometry and autoradiography measurements.

The radioactivity was measured with spectrometry using an Auto Well Gamma System (ARC-500, Aloka, Tokyo, Japan). The results were compared with an $1 \%$ injected dose standard to obtain the tissue uptake rate in terms of the radioactivity percentage of the injected dose per gram of wet tissue (\%dose/g). The uptake corrections were performed based on the blood ${ }^{201} \mathrm{Tl}$ retention rate.

The muscles were embedded in Tissue Tec OCT Compound and frozen in Isopentane pre-cooled in liquid nitrogen. The frozen muscles were crosssectioned at $50 \mu \mathrm{m}$ and dried at $-25^{\circ} \mathrm{C}$. Imaging plates were exposed to the sections inside cassettes within a lead depository to avoid high background radiation. After 2 3 days of exposure, the imaging plates were read by a bio-imaging analyzer (Fuji, BAS 5000, MultiGauge).

Data are expressed as means \pm S.D. (standard deviation) of the number of rats used in each experiment $(n=4)$. One-way ANOVA was used to determine whether the variations among the groups were significant. The muscle uptakes were analyzed by Dunnett's test by comparing experimental groups (UØ, U3w, R10m, R6h, R24h, and R4d) to the control group (C1). R12d and C2 data were compared by a two-tailed, paired t-test. Tukey's test was performed to determine specific differences among muscles in the same group. A value of $\mathrm{p}<0.05$ was accepted as statistically significant. 
Table 1. Mean relative weight: muscle wet weight $(\mathrm{mg}) /$ body weight $(\mathrm{g})(\mathrm{n}=4)$

\begin{tabular}{lccccccccc}
\hline $\begin{array}{l}\text { muscle(mg)/ } \\
\text { body(g) }\end{array}$ & $\mathrm{C} 1$ & $\mathrm{U} \varnothing$ & $\mathrm{U} 3 \mathrm{w}$ & $\mathrm{R} 10 \mathrm{~m}$ & $\mathrm{R} 6 \mathrm{~h}$ & $\mathrm{R} 24 \mathrm{~h}$ & $\mathrm{R} 4 \mathrm{~d}$ & $\mathrm{C} 2$ & $\mathrm{R} 12 \mathrm{~d}$ \\
\hline $\mathrm{Sol}$ & $0.50 \pm 0.03$ & $0.47 \pm 0.02$ & $0.32 \pm 0.06^{* *}$ & $0.38 \pm 0.05^{* *}$ & $0.28 \pm 0.03^{* *}$ & $0.31 \pm 0.03^{* *}$ & $0.43 \pm 0.07$ & $0.44 \pm 0.04$ & $0.46 \pm 0.02$ \\
$\mathrm{Pla}$ & $0.94 \pm 0.05$ & $1.04 \pm 0.05$ & $1.07 \pm 0.11$ & $1.09 \pm 0.05$ & $1.20 \pm 0.54$ & $0.88 \pm 0.08$ & $0.98 \pm 0.10$ & $1.00 \pm 0.06$ & $1.08 \pm 0.07$ \\
$\mathrm{Gas}$ & $5.07 \pm 0.13$ & $4.85 \pm 0.27$ & $5.25 \pm 0.42$ & $4.96 \pm 0.19$ & $5.73 \pm 2.60$ & $4.47 \pm 0.29$ & $4.58 \pm 0.22$ & $5.10 \pm 0.14$ & $5.30 \pm 0.13$ \\
$\mathrm{EDL}$ & $0.48 \pm 0.01$ & $0.50 \pm 0.04$ & $0.56 \pm 0.03$ & $0.59 \pm 0.07$ & $0.65 \pm 0.32$ & $0.48 \pm 0.02$ & $0.50 \pm 0.05$ & $0.49 \pm 0.02$ & $0.52 \pm 0.02$ \\
$\mathrm{TA}$ & $1.85 \pm 0.04$ & $1.92 \pm 0.14$ & $2.10 \pm 0.13$ & $2.18 \pm 0.10$ & $1.66 \pm 0.22$ & $1.77 \pm 0.10$ & $1.87 \pm 0.14$ & $1.91 \pm 0.05$ & $1.91 \pm 0.06$ \\
\hline
\end{tabular}

The means \pm S.D. are shown. Sol: Soleus; Pla: Plantaris; Gas: Gastrocnemius; EDL: Extensor Digitorum Longus; TA: Tibialis Anterior. m: minutes; h: hours; d: days; w: weeks. ** vs. C1 $(\mathrm{p}<0.01)$.

Table 2. Mean uptake rates of ${ }^{201} \mathrm{Tl}$ in skeletal muscles of $\mathrm{C} 1$ (control 1), UØ(at start of unloading), U3w (HU for 3 weeks without reloading), R10m (reloaded for $10 \mathrm{~m}$ after HU), R6h (reloaded for $6 \mathrm{~h}$ after HU), R24h (reloaded for $24 \mathrm{~h}$ after $\mathrm{HU}$ ), R4d (reloaded for 4 days after HU), C2 (control 2), R12d (reloaded for 12 days after HU)

\begin{tabular}{llllll}
\hline Average & Sol & Pla & Gas & EDL & TA \\
\hline C1 & $0.416 \pm 0.03$ & $0.173 \pm 0.03$ & $0.137 \pm 0.01$ & $0.093 \pm 0.02$ & $0.117 \pm 0.02$ \\
UØ & $0.140 \pm 0.03^{* *}$ & $0.210 \pm 0.03$ & $0.197 \pm 0.01 * *$ & $0.247 \pm 0.06^{* *}$ & $0.242 \pm 0.03^{* *}$ \\
U3w & $0.171 \pm 0.02^{* *}$ & $0.104 \pm 0.02^{*}$ & $0.134 \pm 0.03$ & $0.225 \pm 0.05^{* *}$ & $0.199 \pm 0.04^{* *}$ \\
R10m & $0.380 \pm 0.02$ & $0.168 \pm 0.02$ & $0.163 \pm 0.01$ & $0.119 \pm 0.02$ & $0.137 \pm 0.02$ \\
R6h & $0.406 \pm 0.03$ & $0.184 \pm 0.03$ & $0.197 \pm 0.02^{* *}$ & $0.092 \pm 0.02$ & $0.109 \pm 0.01$ \\
R24h & $0.428 \pm 0.07$ & $0.158 \pm 0.03$ & $0.191 \pm 0.02^{* *}$ & $0.098 \pm 0.02$ & $0.129 \pm 0.02$ \\
R4d & $0.504 \pm 0.07 * *$ & $0.154 \pm 0.01$ & $0.181 \pm 0.01 *$ & $0.149 \pm 0.01$ & $0.152 \pm 0.01$ \\
C2 & $0.400 \pm 0.03$ & $0.122 \pm 0.02$ & $0.120 \pm 0.02$ & $0.069 \pm 0.01$ & $0.086 \pm 0.01$ \\
R12d & $0.446 \pm 0.04$ & $0.141 \pm 0.03$ & $0.160 \pm 0.02$ & $0.109 \pm 0.01 \ddagger$ & $0.130 \pm 0.01 \%$ \\
\hline
\end{tabular}

Uptake rates are expressed as percentages of administered dose taken up per gram of wet tissue weight (\%dose/g). The means \pm S.D. for four rat experiments are shown. Sol: Soleus; Pla: Plantaris; Gast: Gastrocnemius; EDL: Extensor Digitorum Longus; TA: Tibialis Anterior. m: minutes; h: hours; d: days; w: weeks. * vs. $\mathrm{C} 1(\mathrm{p}<0.05),{ }^{*}$ vs. $\mathrm{C} 1(\mathrm{p}<0.01)$, tvs. C2 $(\mathrm{p}<0.01)$.

\section{RESULTS}

The muscle relative weights (muscle mass/body mass) are shown in Table 1. The Sol ratio was smaller than the control $(\mathrm{C} 1)$ in $\mathrm{U} 3 \mathrm{w}, \mathrm{R} 10 \mathrm{~m}, \mathrm{R} 6 \mathrm{~h}$, $\mathrm{R} 24 \mathrm{~h}(\mathrm{p}<0.01)$. No statistical differences were found among the other groups. The final mean body weights for C1, UØ, U3w, R10m, R6h, R24h, R4d, $\mathrm{R} 12 \mathrm{~d}$, and C2 were $392.25 \pm 20.4 \mathrm{~g}, 409.25 \pm 39.9$ $\mathrm{g}, 273.5 \pm 25.9 \mathrm{~g}, 260.5 \pm 18.4 \mathrm{~g}, 262.5 \pm 15.5 \mathrm{~g}$, $248.5 \pm 19.7 \mathrm{~g}, 289.5 \pm 11.1 \mathrm{~g}, 348.5 \pm 25.1 \mathrm{~g}$, and $416.75 \pm 8.7 \mathrm{~g}$ respectively.

Table 2 summarizes the mean uptake rates of ${ }^{201} \mathrm{Tl}$ in the 5 hindlimb muscles with HU at two time points (UØ, $\mathrm{U} 3 \mathrm{w})$ and hindlimb reloading at five different time points (R10m, R6h, R24h, R4d, $\mathrm{R} 12 \mathrm{~d})$. The mean \pm standard deviation of four rats is shown for each group. The muscles ${ }^{, 201} \mathrm{Tl}$ uptake rates were corrected according to blood retention values normalized to $0.04 \%$ dose $/ g$. The experimental groups ( $\mathrm{U}$ and $\mathrm{R}$ groups) were compared to the corresponding control group. The Sol ${ }^{201} \mathrm{Tl}$ uptake rates were significantly lower in $\mathrm{U}$ groups $(\mathrm{p}<0.01)$ than in the $\mathrm{C} 1$ group, whereas $\mathrm{R} 4 \mathrm{~d}$ Sol had higher values than $\mathrm{C} 1(\mathrm{p}<0.01)$. The Pla mean uptake in the $\mathrm{U} 3 \mathrm{w}$ group was the lowest among all the groups and was significantly different from the $\mathrm{C} 1$ group $(\mathrm{p}<0.05)$. The Gas uptakes were higher in UØ, R6h, R24h, and R4d than in the control group (C1). U groups' EDL and TA uptakes were higher than those of $\mathrm{C} 1(\mathrm{p}<0.01)$ and EDL and TA of R12d had greater uptake rates than those of C2 $(\mathrm{p}<0.01)$.

Statistical comparisons were also made between muscles' uptake rates of the same group. Sol was the muscle with the highest ${ }^{201} \mathrm{Tl}$ uptake in the $\mathrm{R}$ groups and control groups $(\mathrm{p}<0.01)$. The $\mathrm{C} 1$ group had a Pla mean uptake rate that was greater than those of EDL and TA $(p<0.05)$. Sol of UØ had a lower uptake than either EDL or TA $(p<0.01)$. The U3w group also had high uptake rates in EDL and TA. However, the EDL mean uptake rate in R10m 

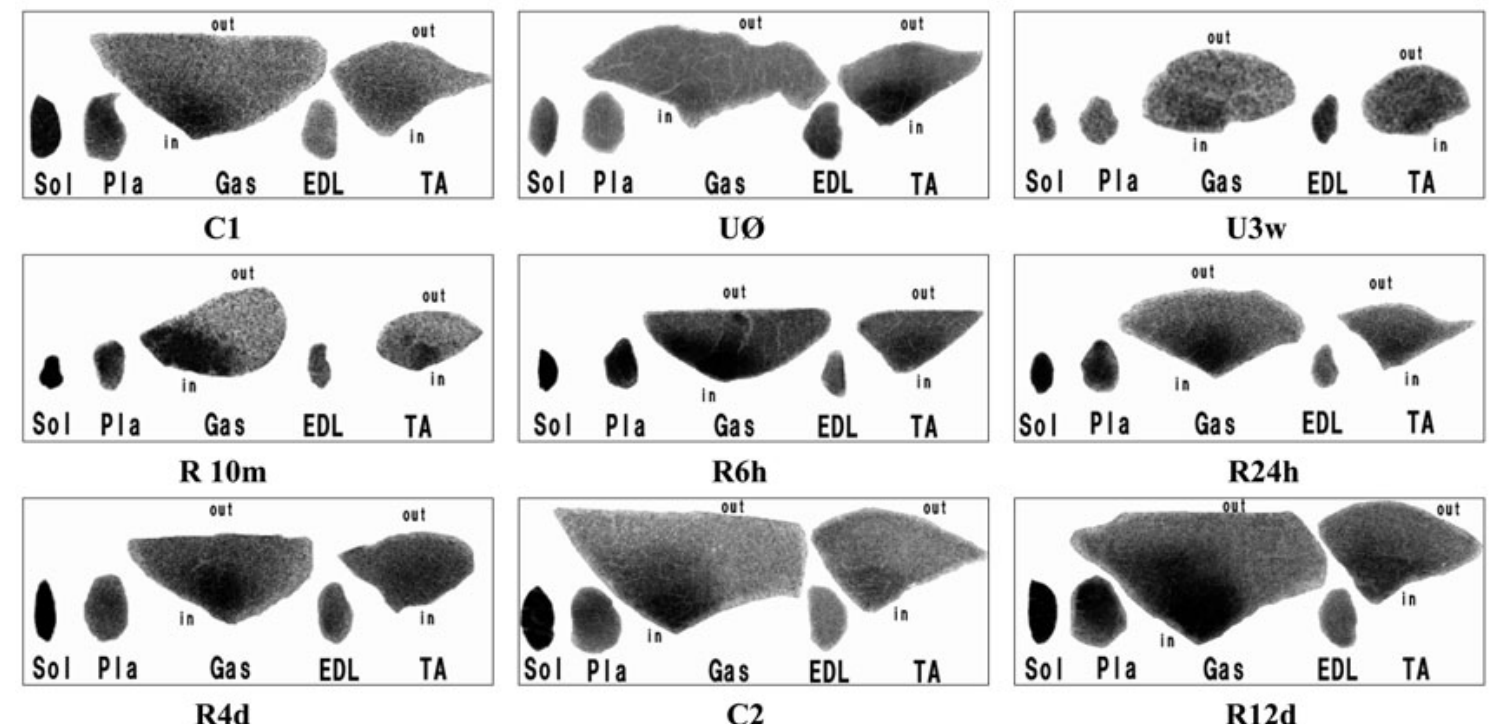

Fig. 1. Hindlimb muscles ${ }^{201} \mathrm{Tl}$ distribution image samples. Sol: Soleus; Pla: Plantaris; Gas: Gastrocnemius; EDL: Extensor Digitorum Longus; TA: Tibialis Anterior; in: inner surface of the muscle; out: outer surface of the muscle.

was the lowest among the control and reloading groups. Pla, Gas, EDL and TA showed no statistical differences among their uptake rates in the R $4 \mathrm{~d}$ and R12d groups.

Figure 1 shows the ${ }^{201} \mathrm{Tl}$ distribution in muscle cross-sections prepared for autoradiography. In the control and reloading groups, ${ }^{201} \mathrm{Tl}$ concentrations are especially proeminent in Sol and the inner layers of Gas and TA.

\section{DISCUSSION}

The Sol mass and body mass ratio, showed marked atrophy after 21 days of HU confirming the effectiveness of the intervention ${ }^{11)}$. In this study, the muscle mass increased rapidly after reloading resulting in no significant differences in relative weights after 4 days of reloading compared to controls.

${ }^{201} \mathrm{TlCl}$ is used to evaluate muscle blood flow by its initial distribution ${ }^{4,5,12}$. The results of this study demonstrate that ${ }^{201} \mathrm{Tl}$ is a very sensitive tracer for evaluating blood perfusion differences between hindlimb muscles.

In comparison to the control group (C1), Sol had lower perfusion immediately after the hindlimb was unloaded (UØ) and also after 3 weeks of HU (U3w). The difference found between Sol of C1 and UØ is probably due to it being an antigravitational muscle, since Sol has an important function and high activity in keeping the posture when the hindlimb is loaded ${ }^{6}$. Muscle blood flow increases or decreases in proportion to the intensity of the activity ${ }^{13)}$ and Riley ${ }^{14)}$ reported that aggregate electromyography of Sol was reduced in hindlimb unloading. ${ }^{201} \mathrm{Tl}$ detected a decrease of Sol blood flow immediately after hindlimb suspension, suggesting a parallel decrease of muscle activity. The hindlimb of the rats in the $\mathrm{U} 3 \mathrm{w}$ group also remained suspended during ${ }^{201} \mathrm{Tl}$ distribution inhibiting the Sol antigravitational activation. However factors other than muscle activity may be involved in low blood perfusion seen in Sol of the U3w group. Kano ${ }^{15)}$ observed in rats' Sol, a significant smaller capillary luminal diameter and lower capillary-to-fiber ratio after 1 week and 3 weeks of HU compared to age-matched control groups.

Intense blood flow was shown by ${ }^{201} \mathrm{Tl}$ in the control and reloading groups. Sol blood flow during reloading is related to many factors. Recovery of muscle fibers from disuse atrophy involves regeneration and repair ${ }^{11)}$. Dumont et al. ${ }^{16)}$ reported that reloading activated mast cells, influenced leukocyte recruitment in the early reloading period ( 1 day and 3 days). The inflammatory response is one possible cause of blood flow increase. Powers ${ }^{17)}$ also related that reloading after HU increased vasodilatory response. McCurdy et 
al. ${ }^{18)}$ concluded that dilatory responsiveness of arterioles varies in muscles composed of different fiber types, and suggested that the inability to adequately elevate peripheral vascular resistance after HU might be due to lowered vasoconstrictor responsiveness and myogenic activity.

Spectrometry can measure the total radioactivity of each muscle sample. However, the ARG imaging revealed detailed distribution of ${ }^{201} \mathrm{~T} 1$. This technique can be used to detect the heterogeneity of blood perfusion in the muscle ${ }^{10)}$, that is related to muscle fiber type distribution. The concentration of ${ }^{201} \mathrm{Tl}$ in the inner layers of Gas and TA may be related to Type I muscle fiber regionalization. Wang ${ }^{19)}$ observed that type I fibers were typically greatest towards the center of the hindlimb in rats.

We conclude that during 21 days of HU and subsequent recovery by reloading, blood capillary perfusion as assessed by ${ }^{201} \mathrm{Tl}$ in hindlimb muscles changes in rats. ${ }^{201} \mathrm{Tl}$ is an available, safe and easy method already widely used in human medicine to evaluate myocardium and tumors. The results of the present study confirm the utility of ${ }^{201} \mathrm{~T} 1$ in quantitative and, by imaging, qualitative evaluation of skeletal muscle activity. ${ }^{201} \mathrm{Tl}$ provides a detailed assessment of blood perfusion, with applications in rehabilitation and sports science. Further investigations are needed to clarify and prove the efficacy of ${ }^{201} \mathrm{Tl}$ in the evaluation of skeletal muscle diseases.

\section{ACKNOWLEDGEMENT}

This work was supported by a Grant-Aid for Young Scientists (Start-up) from KAKENHI (20800021) of JSPS.

\section{REFERENCES}

1) Saha GB, Boyd CM: Miscellaneous Radiotracers for Imaging. In: Radiotracers for Medical Applications. Florida: CRC Press, 1983, pp119-132.

2) Sessler MJ, Geck P, Maul FD, et al.: New aspects of cellular thallium uptake: $\mathrm{Tl}^{+}-\mathrm{Na}^{+}-2 \mathrm{Cl}\left({ }^{-}\right)$-cotransport is the central mechanism of ion uptake. Nucl Med, 1986, 25: 24-27.

3) Travin MI, Bergmann SR: Assessment of Myocardial Viability. Semin Nucl Med, 2004, 9: 2-16.

4) Christenson J, Larsson I, Svensson S-E, et al.: Distribution of intravenously injected ${ }^{201}$ thallium in the legs during walking: A new test for assessing arterial insufficiency in the legs. Eur J Nucl Med,
1977, 2: 85-88.

5) Oshima M, Yano $T$, Nishikimi $\mathrm{N}$, et al.: Leg muscle perfusion study using Tl-201 single photon emission computed tomography (SPECT). Kaku Igaku, 1986, 23 (2): 153-159.

6) Yamazaki $T$, Haida $\mathrm{N}$, Tachino $\mathrm{K}$ : Influence of the time when weight bearing is started on disuse atrophy in rat soleus muscle. J Jpn Phys Ther Assoc, 2001, 4: 13-18.

7) Thomason DB, Booth FW: Atrophy of the soleus muscle by hindlimb unweighting. Am J Physiol, 1990, 161: 7567-7590.

8) Yamazaki T, Haida N, Tachino K: Effect of weightbearing frequency per day in retarding disuse atrophy in rat soleus muscle. Rigaku Ryoho Journal, 1996, 30: 53-57.

9) Inaoka PT, Tachino K, Ryohei A, et al.: Uptake behavior of Thallium-201 in hindlimb muscles of rats. J Phys Ther Sci, 2008, 20(2): 117-122.

10) Mizuno M, Kimura $Y$, Iwakawa $T$, et al.: Regional differences in blood flow and oxygen consumption in resting muscle and their relationship during recovery from exhaustive exercise. J Appl Physiol, 2003, 95: 2204-2210.

11) Itai $Y$, Kariya $Y$, Hoshino $Y$ : Morphological changes in rat hindlimb muscle fibres during recovery from disuse atrophy. Acta Physiol Scand, 2004, 181: 217224.

12) Brismar T: In vivo analysis of intracellular thallium201 accumulation in skeletal muscle of the rat. Acta Physiol Scand, 1991, 142: 475-480.

13) Thomas GD, Segal SS: Neural control of muscle blood flow during exercise. J Appl Physiol, 2004, 97: 731738.

14) Riley DA, Slocum GR, Bain JLW, et al.: Rat hindlimb unloading: soleus histochemistry, ultrastructure, and electromyography. J Appl Physiol, 1990, 69(1): 5866.

15) Kano Y, Shimegi S, Takahashi H, et al.: Changes in capillary luminal diameter in rat soleus muscle after hind-limb suspension. Acta Physiol Scand, 200, 169: 271-276

16) Dumont $\mathrm{N}$, Lepage $\mathrm{K}$, Côté $\mathrm{CH}$, et al.: Mast cells can modulate leukocyte accumulation and skeletal muscle function following hindlimb unloading. J Appl Physiol, 2007, 103: 97-104.

17) Powers J, Bernstein D: The mouse as a model of cardiovascular adaptations to microgravity. J Appl Physiol, 2004, 97:1686-1692.

18) McCurdy MR, Colleran PN, Muller-Delp J, et al.: Physiology of a microgravity environment selected contribution: Effects of fiber composition and hindlimb unloading on the vasodilator properties of skeletal muslcle arterioles. J Appl Physiol, 2000, 89: 398-405.

19) Wang LC, Kernell D: Quantification of fibre type regionalisation: an analysis of lower lindlimb muscles in the rat. J Anat, 2001, 198: 295-308. 